\title{
POPULATIONS AMONG HIGH-VELOCITY EARLY-TYPE STARS
}

\author{
F. ROYER, A.E. GÓMEZ \\ DASGAL URA 335, Observatoire de Paris-Meudon, F-92195 Meudon
}

As far as the standard picture of galactic evolution and structure is concerned, early-type stars belong to disk populations. However, the existence of a population of early-type stars with nearsolar metallicities and main-sequence surface gravities, together with high velocities and/or standing at large distances away from the plane, is a well known anomaly to this picture.

In order to provide an insight into the different populations of early-type stars, a sample of high-velocity stars has been gathered from the Hipparcos catalogue. We have selected the stars according to $B-V<0.4$, and a tangential velocity larger than $80 \mathrm{~km} / \mathrm{s}$. The sample is composed of 101 stars.

The high-velocity early-type stars in the solar neighbourhood are a mixture of different stellar populations : blue horizontal halo stars passing near the sun and high-velocity disk stars. We have decomposed into gaussians the $V$ and $W$-distributions of our sample, to isolate the disk from the halo populations. The $U$-distribution of high-velocity disk stars is clearly asymmetric. The selection bias over the tangential velocities creates a depletion of low $U$ values. Moreover, two thirds of the sample have $U<0$ (toward the galactic anticentre, $l=180^{\circ}$ ). This cannot be explained by the distribution tail of disk stars or stochastic events such as runaway stars, which privilege no direction.

\section{A SEARCH FOR STARS PASSING CLOSE TO THE SUN}

\author{
J. GARCIA-SANCHEZ, R.A. PRESTON, D.L. JONES, P.R. WEISSMAN \\ JPL, California Institute of Technology,Pasadena, CA 91109, USA \\ J.-F. LESTRADE \\ Observatoire de Paris-Meudon, F-92195 Meudon Principal Cedex, France \\ AND \\ D.W. LATHAM AND R.P. STEFANIK \\ Harvard-Smithsonian CfA, Cambridge, MA 02138, USA
}

We have combined radial velocity measurements with Hipparcos proper motion and parallax data for 552 nearby stars of a selected sample of 1208 stars to find which stars may have passed (or will pass) close enough to the Sun to perturb the cometary Oort cloud. We find that the rate of close stellar approaches within a distance $D$ (in pc) from the Sun is $\mathrm{N}=4.3 \mathrm{D}^{2.02} \mathrm{Myr}^{-1}$, in excellent agreement with the predicted functional form of simple stellar dynamics models though the number of stars is less than predicted. However, we consider this a lower limit because there is evidence for observational incompleteness in our data set. One star (Gliese 710) is found with a future closest approach of less than $0.4 \mathrm{pc}$, and several stars come within about $1 \mathrm{pc}$ during about a $\pm 10 \mathrm{Myr}$ interval. We have performed dynamical simulations which show that none of the passing stars in this sample can perturb the Oort cloud sufficiently to create a substantial increase in the long-period comet flux at the Earth's orbit. 